\title{
Urban Traffic Noise Maps under 3D Complex Building Environments on a Supercomputer
}

\author{
Ming Cai $\mathbb{D}^{1,2,3}$ Yifan Yao $\mathbb{D}^{1,2,3}$ and Haibo Wang $(\mathbb{D})^{1,2,3,4}$ \\ ${ }^{1}$ School of Intelligent Systems Engineering, Sun Yat-sen University, Shenzhen, China \\ ${ }^{2}$ Guangdong Provincial Key Laboratory of Intelligent Transportation System, Guangzhou, China \\ ${ }^{3}$ Guangdong Provincial Engineering Research Center for Traffic Environmental Monitoring and Control, Guangzhou, China \\ ${ }^{4}$ School of Civil and Transportation Engineering, Hebei University of Technology, Tianjin, China
}

Correspondence should be addressed to Haibo Wang; wanghb9@mail.sysu.edu.cn

Received 13 April 2018; Revised 12 June 2018; Accepted 25 June 2018; Published 9 July 2018

Academic Editor: Konstantinos Vogiatzis

Copyright (c) 2018 Ming Cai et al. This is an open access article distributed under the Creative Commons Attribution License, which permits unrestricted use, distribution, and reproduction in any medium, provided the original work is properly cited.

\begin{abstract}
The complexity of the 3D buildings and road networks gives the simulation of urban noise difficulty and significance. To solve the problem of computing complexity, a systematic methodology for computing urban traffic noise maps under 3D complex building environments is presented on a supercomputer. A parallel algorithm focused on controlling the compute nodes of the supercomputer is designed. Moreover, a rendering method is provided to visualize the noise map. In addition, a strategy for obtaining a real-time dynamic noise map is elaborated. Two efficiency experiments are implemented. One experiment involves comparing the expansibility of the parallel algorithm with various numbers of compute nodes and various computing scales to determine the expansibility. With an increase in the number of compute nodes, the computing time increases linearly, and an increased computing scale leads to computing efficiency increases. The other experiment is a comparison of the computing speed between a supercomputer and a normal computer; the computing node of Tianhe- 2 is found to be six times faster than that of a normal computer. Finally, the traffic noise suppression effect of buildings is analyzed. It is found that the building groups have obvious shielding effect on traffic noise.
\end{abstract}

\section{Introduction}

With rapid growth of population and the continued expansion of transportation systems, traffic noise pollution becomes quite a nuisance to urban residents. In Europe, the recent Environmental Noise Directive (END) revision has updated the current situation on the application of the END [1] and noise pollution continues to be a major health problem. The growing amount of noise pollution can lead to a number of serious diseases, including sleep disturbance $[2,3]$, stroke [4], male infertility [5], and learning impairment [6]. Moreover, long-term exposure to traffic noise can easily lead to high blood pressure [7] and affect the quality of life in the neighborhood [8].

Since END 's prescription for noise maps and action plans, there have been many efforts by the scientific community to conduct high-level study and propose new mitigation systems for the main sources of noise in urban areas: road traffic, railway traffic, airport, and industrial, ranging from regulations to operational approaches [9-11]. A noise map is an important tool for observing and controlling noise pollution and the basis for epidemiology study relating annoyance to noise and noise exposure in urban areas. In urban planning, a noise map is used to analyze the sound qualities of the soundscapes in a specific urban area to generate recommendations for the urban design of the soundscapes [12]. For example, Palma de Mallorca (Spain) conducted a study analyzing various noise mitigation measures, which consider not only the reduction of noise and the number of people who can benefit from these measures but also the net monetary benefits generated, by using a traffic noise map [13]. Another study proposed a method for sorting road stretches by priority by using a noise map. The method is based on the so-called "road stretch priority index", in which the index involves a number of variables that are weighted according to their influence on the road traffic noise problem, and 
road stretches of different priorities thus require different plan actions [14]. Similarly, Daniel Naish proposed a regional road traffic noise management strategy (RRTNMS); in this strategy, the road was ranked according to the predicted sound pressure level, and different control measures were implemented according to the results of the ranking [15]. In both studies, noise maps play a key role in the prediction of traffic noise and the display of final result. Furthermore, noise impact indicators and the mean of the expected individual annoyance scores in the population are calculated on the basis of a noise map. Those indicators include the percentages of people being highly annoyed, annoyed, and slightly annoyed and are used to compare the effect of noise reduction [16].

The research on noise maps started with the publication of END; since then, many scholars have researched noise maps, covering the calculating model and the update method for a noise map [17-23]. The calculation of a noise map is a complex process that involves a traffic noise prediction model and sound propagation attenuation. Nicolas Fortin et al. implemented a noise prediction method within the OrbisGIS2 software [24]; the method can produce large noise maps on a personal computer in a few hours, but the noise map is two-dimensional rather than three-dimensional. A smallscale 3D noise map can be implemented well by combining the prediction model and measured data to obtain a highprecision noise map [25]. However, since the complexity of the noise map calculation is the square of the computing scale which includes receiver point's density and map size, a largescale 3D noise map requires an excessive amount of time when using a normal computer with a CPU containing no more than 16 computing cores (the normal computer information used in the experiments in this article is described in Section 9). A study in 2009 considered computing a noise map on a supercomputer; in this effort, software called Noise Propagation Model is used to obtain the noise map in a supercomputer [26]; the parallel algorithm assigns tasks at one time rather than using dynamic assignment, and the noise map is two-dimensional. In addition, a traffic noise map was computed in the cloud computing environment [27]; however, in that study, the noise map produced was an existing service in the cloud computing system, and the computational framework was not explained.

In the paper, a method for computing a large-scale noise map in three dimensions on a supercomputer is presented to solve the problem of time-consuming calculation of dynamic three-dimensional noise maps. The supercomputer used in this study is Tianhe-2, and a computation strategy using Tianhe-2 is introduced and its performance is analyzed. The entire noise map calculation process includes a noise prediction model, a parallel calculation algorithm, and a visualization scheme of noise map. Then, combined with taxi trajectory data, a real-time noise map implementation method was proposed to dynamically update the noise map of the entire city. In addition, two experiments were carried out to analyze the efficiency of the parallel algorithm, one is about the expansibility analysis of the noise map parallel computing algorithm proposed in this paper, and the other is the analysis of the computing efficiency of Tianhe-2. Finally, we analyze the suppression effect on traffic noise of buildings from the changes in noise distribution on the ground and building surface caused by building groups.

\section{Traffic Noise Prediction among Building Groups}

Noise map is composed of noise receiver points and it reflects the noise distribution in an urban area. Vehicles running on the road generate noise that propagates across buildings in a city before reaching the receiver point. Therefore, a model for predicting traffic noise has three steps: the first step is to predict the acoustical power of road, the second step is to predict sound attenuation caused by the building group in an urban area, and the last step is to combine all of the road's acoustic power with the sound attenuation caused by the building group to calculate the noise value of the noise receiver point [28].

The factors affecting road noise emissions include traffic volume, road speed, and road surface materials. Among them, road surface materials are inherent attributes of roads, and good road surface materials can effectively reduce road noise emissions $[29,30]$.This paper is based on China's road environment. China's roads are basically asphalt pavements, so the impact of pavement materials as a fixed item is not reflected in the model parameters. As a result, the acoustical power of road is calculated using (1) and (2):

$$
\begin{aligned}
L_{e q \mathrm{i}} & =L_{o i}+10 \lg \frac{N_{i}}{T V_{i}}+10 \lg \left(\frac{r_{0}}{r}\right)+10 \lg \left(\frac{\psi}{\pi}\right)-16 \\
L_{e q} & =10 \lg \left(10^{0.1 L_{\text {eqs }}}+10^{0.1 L_{\text {eqm }}}+10^{0.1 L_{\text {eql }}}\right)
\end{aligned}
$$

where $L_{e q i}$ is equivalent sound level of the ith type traffic flow to the receiver point without shelter, $L_{e q}$ is Equivalent sound level of the road to the receiver point without shelter, $L_{o i}$ is average sound level of the ith type traffic flow to the reference point, $N_{i}$ is traffic flow volume of the ith type traffic flow, $V_{i}$ is average speed of the ith type traffic flow, $T$ is the prediction time, $r_{0}$ is distance between the reference point and the lane $\left(r_{0}=7.5 \mathrm{~m}\right), r$ is distance between the receiver point and the lane, and $\psi$ is the field angle formed by the receiver point, the origin point of the road, and the end point of the road, with the peak of the angle being the receiver point.

In the model, the sound attenuation caused by the building group has two parts:

$$
A_{\text {building }}=A_{\text {building } 1}+A_{\text {building } 2}
$$

$A_{\text {building1 }}$ denotes the immediate shelter effect on the propagation path of sound caused by building group, and the relevant prediction equation is

$$
A_{\text {building } 1}=0.1 C d_{b}
$$

where $C$ is the density of the building group on the propagation path of sound and $d_{b}$ is the length of the sound propagation path.

$A_{\text {building2 }}$ is the shelter effect of sound diffusion caused by the building group, and the relevant prediction equation is

$$
A_{\text {building } 2}=-10 \lg (1-p)
$$


where $p$ is the ratio of the projection length on the road of buildings near the road divided by the road length.

In total, the noise value of the receiver point should combine all roads and the attenuation of those roads:

$$
L_{A}=10 \lg \sum_{k} 10^{0.1\left(L_{e q}^{k}-A_{\text {building }}^{k}\right)}
$$

where $L_{A}$ is the equivalent sound level of receiver point $A$, $L_{e q}^{k}$ is the equivalent sound level of road $k$ to a receiver point without shelter, and $A_{\text {building }}^{k}$ is the sound attenuation caused by the building group between road $k$ and the receiver point.

\section{Parallel Algorithm in a Supercomputer}

Urban areas contain a large number of buildings with complicated layouts and irregular outlines, which increases the computation complexity of a noise map, especially a threedimensional one. However, simplifying the prediction model would cause the noise map to have low prediction accuracy. In this condition, a supercomputer is applied to compute a largescale 3D noise map. In this study, Tianhe- 2 is used to compute a noise map in three dimensions. Tianhe- 2 was announced in June 2013, with Intel Xeon Phi accelerators contributing more than $85 \%$ of the 55 pflops peak performance [31]. The strong computing ability of Tianhe- 2 supports the computation of a large-scale 3D noise map on the basis of its hardware; thus, it is critical to have an appropriate and efficient parallel algorithm. The parallel algorithm presented here can be divided into two sections: one is the map tiling, which is the structural basis of parallel computation, and the other section is the coordination of the compute nodes, which implements the computation of noise map.

3.1. Map Tiling. When the distance between the sound source and the receiver point is sufficiently large, the impact of the sound source to the receiver point is so small that it can be ignored. Therefore, breaking up the noise map into a tiling map composed of many smaller rectangle blocks is a reasonable approach for calculating the noise.

The map is tiled into a set of rectangle blocks that are 200 meters in length and 200 meters in width. In each block, the distance between noise receiver points is always set as $4 \mathrm{~m}$, so there are always 2500 receiver points in a block. While computing the noise of a block, the roads and buildings in a wider area are brought into such a state that each receiver point in the block can receive all impacts of the roads near it. Range of the area is determined by the number of roads inside it. As shown in Figure 1, when computing block 0, the roads and buildings in the grey area are also brought into the computation. The initial size of the grey area is $600 \mathrm{~m} \times 600 \mathrm{~m}$, and it gradually expands until the number of roads inside it is larger than a threshold $\theta$.

3.2. Coordination of the Compute Nodes. Because a supercomputer is composed of a large number of compute nodes and the map is tiled into a set of blocks, one compute node can compute one block at a time. As a result, $\mathrm{N}$ blocks can be computed simultaneously if $\mathrm{N}$ compute nodes are

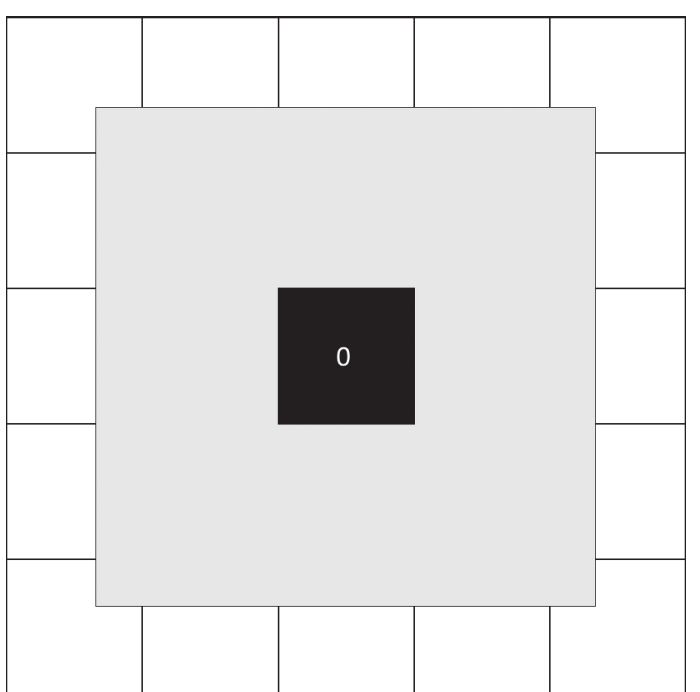

FIGURE 1: Take eight neighborhoods of block 0 into the computation.

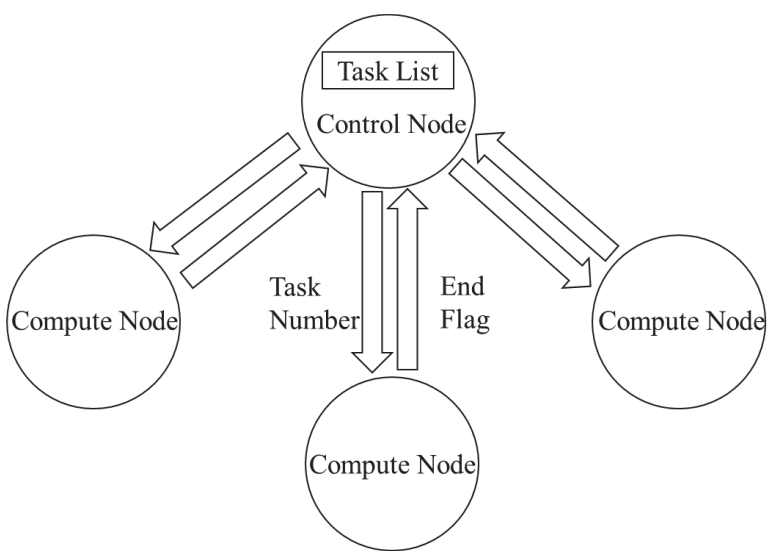

FIGURE 2: The process of parallel control on a supercomputer.

applied to compute the noise map. The critical point lies in coordinating those compute nodes. In the algorithm, one node is specifically used to control the computation of the other nodes. In the control node, a task list is running to assign tasks to the compute nodes. The control node sends the task number to the compute node, which represents the block index that the compute node is supposed to compute, and the compute node sends the end flag to the control node when the task is finished; the process is shown in Figure 2.

To maximize the computational efficiency and reduce the idle time of compute node, the control logic of control node is supposed to be dynamic. This process requires that whenever a compute node completes its task, the control node assigns a new task for it. The control logic is shown in Figure 3. First, the control node assigns the first task for each compute node, and the control node turns into the loop state. In the loop state, the control node checks whether any compute node has completed its computations and then sends an end flag to that compute node. Whenever the control node finds an idle compute node, it sends a new task to the idle compute node until all tasks are completed. 


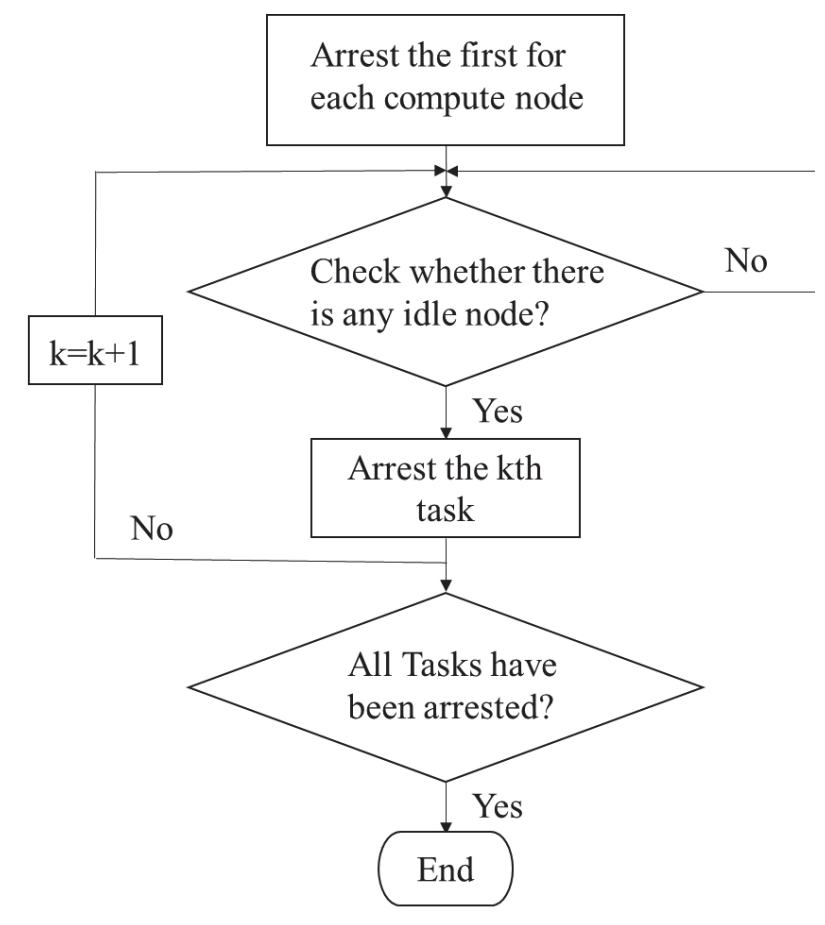

FIGURE 3: Control logic of the control node on a supercomputer.

\section{Rendering a 3D Noise Map}

After computing a noise map on the supercomputer, the noise data must be rendered to visualize the result. It is difficult to render a large-scale noise map without any simplification process. One of the most convenient and rapid methods of simplification is to partly render the noise map according to the current zoom level. The basis of rendering the noise map is the conversion of a noise value into a color value.

4.1. Render in Grading. The zoom level determines the range of noise map to be rendered; thus, the noise receiver points required to render the noise map can be selected at a certain distance, and not all of the receiver points must be used in the rendering if the zoom level is small and the rendering range is large. The distance depends on the current zoom level:

$$
\delta=k \lambda
$$

where $\delta$ is the distance between the noise receiver points selected to render the noise map, $k$ is the proportionality coefficient, and $\lambda$ is the current zoom level.

In addition, the receiver point includes the point of a building face; thus, the rendering of the noise distribution in buildings is required. The rendering of the noise distribution in buildings depends on the zoom level as well, and buildings are rendered when the zoom level is greater than a given threshold:

$$
\lambda \geq \lambda_{0}
$$

where $\lambda_{0}$ is the threshold of the zoom level.
4.2. Noise Conversion to Color. The noise map is composed of a variety of receiver points. Each receiver point has a noise value after computing, and visualization requires the conversion from noise to color. Color is expressed by an RGB vector: a noise value larger than $80 \mathrm{~dB}$ corresponds to red, whose RGB vector is $(1,0,0)$, a noise value less than $40 \mathrm{~dB}$ corresponds to green, whose RGB vector is $(0,1,0)$, and a noise value between $40 \mathrm{~dB}$ and $80 \mathrm{~dB}$ is calculated as

$$
\mathrm{R} G B=\left(\frac{(\text { Noise }-40)}{40}, \frac{(80-\text { Noise })}{40}, 0\right)
$$

where Noise is the noise value of receiver point.

4.3. Display of the Rendering of Noise Map. The traffic noise rendering result is displayed in Figure 4. Figure 4(a) is at a small zoom level that is not in excess of the threshold, so the building is not rendered. Figure 4(b) is at a zoom level that is in excess of the threshold, so the building is rendered. Figure 4(c) shows the noise distribution on the building face in detail. The green area indicates that the area is very quiet, the red area indicates that the level of noise pollution is serious, and the yellow area indicates that the area is at a normal noise level.

\section{Strategy for Obtaining a Dynamic Noise Map}

The dynamic noise map can reflect the real-time noise pollution and is an extremely effective tool for noise monitoring and implementing a short-term noise prevention plan. Dynamap project has put forward the aim for realizing a real-time noise map, it focuses on the research of a technical solution able to ease and reduce the cost of noise map through an automatic monitoring system [32]. Another project called Noise Tube proposes measuring and mapping noise pollution through public mobile phones [33]. This section proposes a method for drawing dynamic noise maps based on taxi trajectory data and supercomputers to effectively solve the problems of acquiring real-time traffic data and excessive time-consuming when computing noise maps.

5.1. Real-Time Traffic Data. There are many taxis in every city, the tracking information of which is owned by those taxi enterprises. This information is available in real-time. In this study, the traffic data used to predict noise are calculated from the tracking information of almost 20000 taxis in Guangzhou. The instantaneous speed of the taxi is inferred from the real-time location information of the taxi; then, the speed of each taxi on the same road over one hour is averaged to obtain the hourly average speed of the road. Studies have shown that road traffic volume and average road speed have different degrees of influence on traffic noise, and there is a clear correlation between them [34].To obtain the hourly traffic volume, large amounts of traffic monitor video are used to determine the pattern between the road speed and the road traffic volume for different road types, and the hour traffic volume is determined from the hourly average speed. Figure 5 shows the taxi tracking data in Guangzhou, as visualized by 


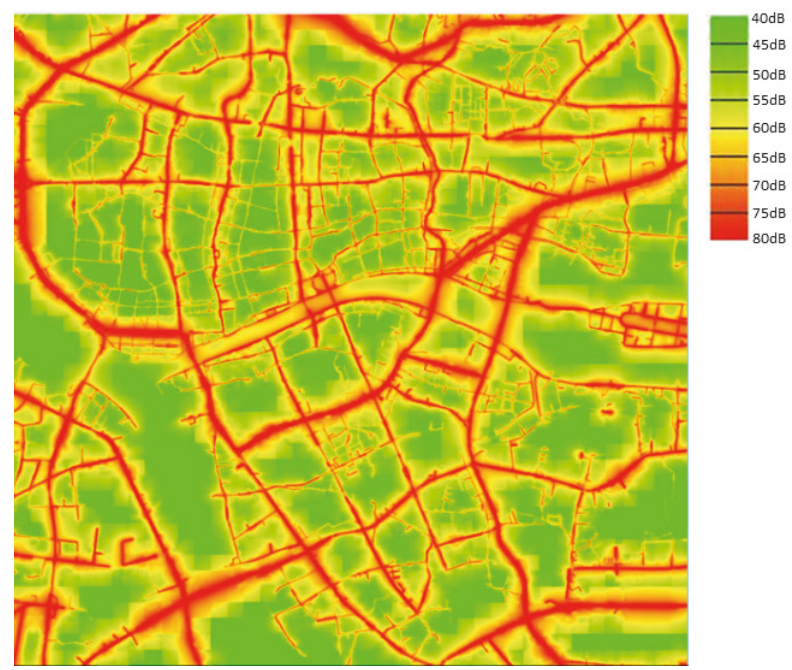

(a) Noise map when the zoom level is less than the threshold

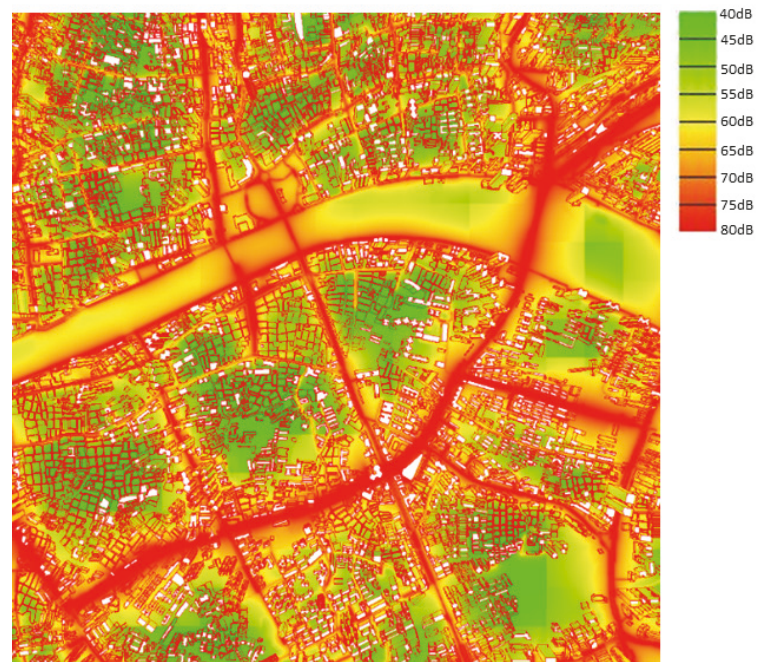

(b) Noise map when the zoom level is greater than the threshold

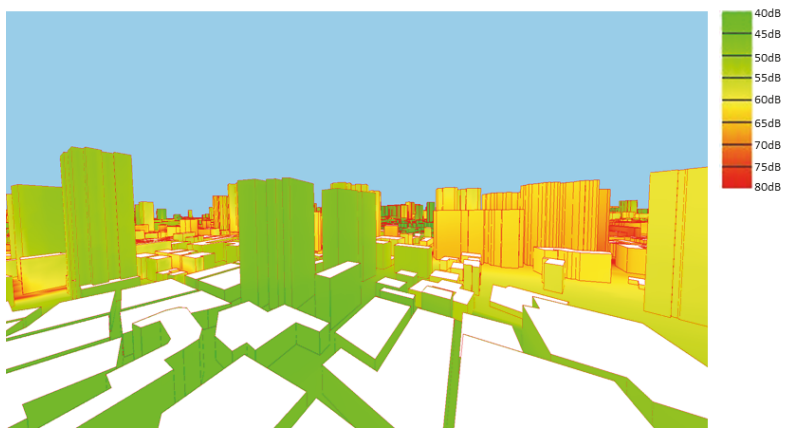

(c) Noise distribution on the building faces

FIgURE 4: 3D noise map calculated by a supercomputer.

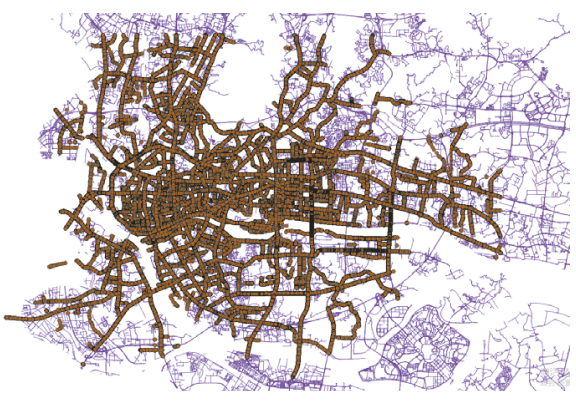

FIgURE 5: Real-time taxi tracking data in Guangzhou.

a GIS tool. Through this method, real-time traffic data does not need to be obtained through all-day traffic monitoring, and traffic data of the entire city can be obtained; accordingly, this method is not precise enough to obtain traffic data with high accuracy.

5.2. Offline Computing. Computing a traffic noise map for the main urban area of Guangzhou on Tianhe- 2 while using 300 compute nodes still requires 22 hours, so an optimization measure is still required to increase the computational speed and obtain a dynamic traffic noise map. It is unreasonable to use more compute nodes to realize the dynamic noise map because doing so would be too expensive. Fortunately, considering the computing process, the sound attenuation caused by the building group $A_{\text {building }}$ is correlated only to the position of the road and buildings according to (4) and (5). Therefore, offline computing is possible for $A_{\text {building }}$ [35].

In the first computation of noise map, $A_{\text {building }}$ is calculated using (4) and (5), and the result is saved in the database. In the following calculation of the noise map, $A_{\text {building }}$ is read from the database rather than calculated using (4) and (5). The offline computing of the sound attenuation caused by the building group improves the computational efficiency vastly, requiring only approximately 222 seconds to compute the traffic noise map in the main urban area of Guangzhou on Tianhe-2 when using 300 compute nodes; thus, there is enough surplus time to generate traffic data from the taxi data.

\section{Efficiency Analysis}

The computation of a large-scale 3D traffic noise map is quite vast, and the computing time is determined by the computing efficiency. To evaluate whether the methodology in the paper 
is feasible, the efficiency is analyzed in this section. The efficiency analysis is divided into two parts: the algorithm and the hardware. The first part is the expansibility of the algorithm, and the second part is the computing efficiency of the supercomputer.

6.1. Expansibility of the Algorithm. The expansibility of algorithm refers to the variation of algorithm's efficiency with a change in the computing resources and the scale of computation. In the experiment, the efficiency of the algorithm is indicated by the computing time, the computing resource is indicated by the number of compute nodes, and the scale of computation is indicated by the step length of the receiver points (in the same map, the smaller the step length is, the greater the number of receiver points is).

The computing time of a noise map is composed of two parts: one is the time for initialization, which lapses at the beginning of the operation of each node, and the other part is the time for computing the blocks. Because the parallel algorithm allows the compute node to be running constantly until all tasks are completed, the computing time of a noise map is similar to the running time of each compute node. From the discussion above,

$$
N_{\text {node }} \times t_{\text {initial }}+N_{\text {block }} \times t_{\text {block }}=T_{\text {actual }} \times N_{\text {node }}
$$

After simplification,

$$
t_{\text {initial }}+\left(\frac{N_{\text {block }}}{N_{\text {node }}}\right) \times t_{\text {block }}=T
$$

where $t_{\text {initial }}$ is the time for initialization of each compute node, $t_{\text {block }}$ is the time for computing each block, $N_{\text {node }}$ is the number of compute nodes, $N_{\text {block }}$ is the number of blocks, and $T_{\text {actual }}$ is actual computing time of the noise map.

The noise map used to measure the expansibility of the algorithm contains 127 road segments and 102 buildings, and the area is $1332 m \times 1785 m$; thus, the noise map is tiled into $7 \times$ 9 blocks. The number of compute nodes and the step lengths of receiver point are changed to measure the expansibility of the algorithm, as indicated in Table 1. In addition, the threshold $\theta$ in map tiling is set as $9 \times N_{\text {block }} / N_{\text {node }}$.

$t_{\text {initial }}$ and $t_{\text {block }}$ in (11) are estimated from Table 1 using the least squares method, and when different numbers of compute nodes are used, $t_{\text {initial }}$ and $t_{\text {block }}$ have different values. The fitting results using the least squares method are displayed in Figure 6, where Ratio represents $N_{\text {block }} / N_{\text {node }}$.

In Figure 6, the abscissa is $N_{\text {block }} / N_{\text {node }}$, and the ordinate is the computing time $T_{\text {actual }}$; the step length of the receiver point varies from $10 \mathrm{~m}$ to $2 \mathrm{~m}$. In the five regression formulas, the slope represents time for computing each block $t_{\text {block }}$, and the intercept represents the time required for the initialization of each compute node $t_{\text {initial }}$.

Equation (11) reveals that the computing time is composed of two parts: the initial time $t_{\text {initial }}$ and the average computing time of each block $t_{\text {block }}$. A specific value between the times is correlated to the efficiency of the algorithm, as shown in Table 2 for a computation using 3 compute nodes.

The following three conclusions can be drawn from the experiment implemented above:

\begin{tabular}{|c|c|c|}
\hline $\begin{array}{l}\text { Step length between } \\
\text { receiver points /m }\end{array}$ & $\begin{array}{l}\text { Number of compute } \\
\text { nodes }\end{array}$ & Computing time $/ \mathrm{s}$ \\
\hline \multirow{5}{*}{10} & 1 & 201 \\
\hline & 2 & 109 \\
\hline & 3 & 73 \\
\hline & 4 & 61 \\
\hline & 5 & 53 \\
\hline \multirow{5}{*}{8} & 1 & 310 \\
\hline & 2 & 160 \\
\hline & 3 & 113 \\
\hline & 4 & 94 \\
\hline & 5 & 77 \\
\hline \multirow{5}{*}{6} & 1 & 538 \\
\hline & 2 & 286 \\
\hline & 3 & 199 \\
\hline & 4 & 169 \\
\hline & 5 & 140 \\
\hline \multirow{5}{*}{4} & 1 & 1204 \\
\hline & 2 & 635 \\
\hline & 3 & 444 \\
\hline & 4 & 368 \\
\hline & 5 & 312 \\
\hline \multirow{5}{*}{2} & 1 & 4766 \\
\hline & 2 & 2568 \\
\hline & 3 & 1821 \\
\hline & 4 & 1480 \\
\hline & 5 & 1260 \\
\hline
\end{tabular}

TABLE 1: Expansibility of algorithm.

TABLE 2: Specific value between $t_{\text {block }}$ and $t_{\text {initial }}$.

\begin{tabular}{lccc}
\hline Step length & $t_{\text {block }} / \mathrm{s}$ & $t_{\text {initial }} / \mathrm{s}$ & $t_{\text {block }} / t_{\text {initial }}$ \\
\hline 10 & 2.9688 & 13.988 & 0.212 \\
8 & 4.61 & 18.169 & 0.254 \\
6 & 7.8935 & 39.305 & 0.201 \\
4 & 17.755 & 81.783 & 0.217 \\
2 & 69.654 & 375.05 & 0.186 \\
\hline
\end{tabular}

(a) Equation (11) is fitted quite well when using the least squares method from Figure 5; all coefficients of the association approach 1 very well, indicating that the computing time is explicitly revealed by (11).

6.2. Computing Efficiency of the Supercomputer. Nearly 22 hours is required to calculate the noise map of the main city zone in Guangzhou on supercomputer when using 300 compute nodes. Thus, an experiment to determine the computing efficiency of a supercomputer is implemented to illustrate the necessity of using a supercomputer to compute a large-scale noise map.

A normal computer with a quad-core CPU and $16 \mathrm{~GB}$ random-access memory is used for comparison with the 


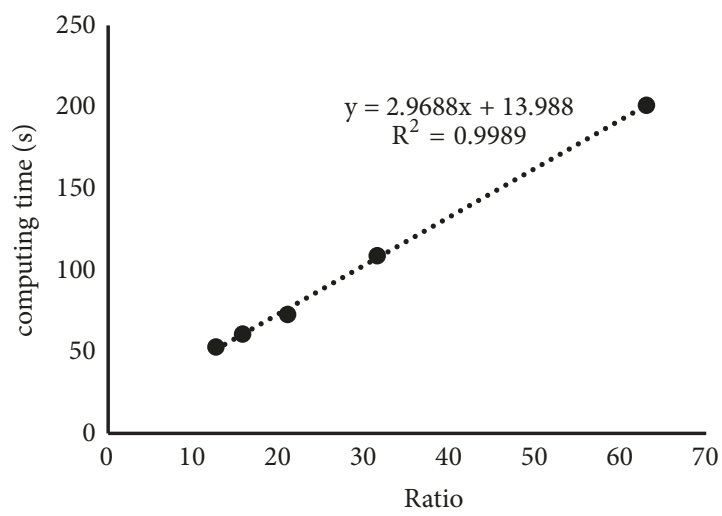

(a) Step length: $10 \mathrm{~m}$

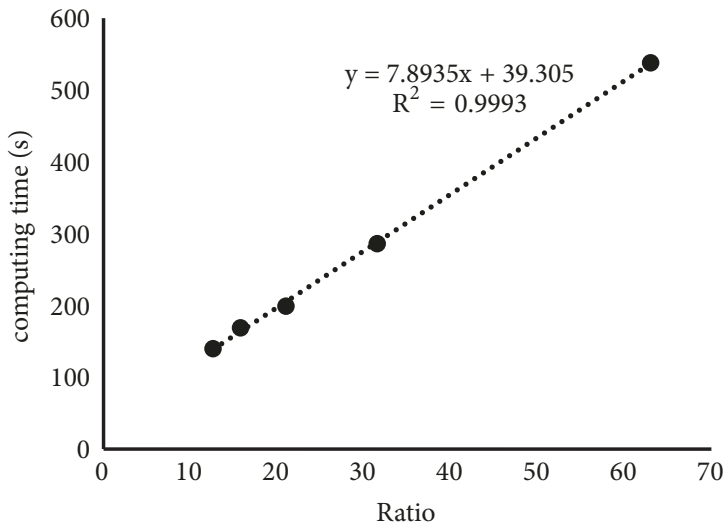

(c) Step length: $6 \mathrm{~m}$

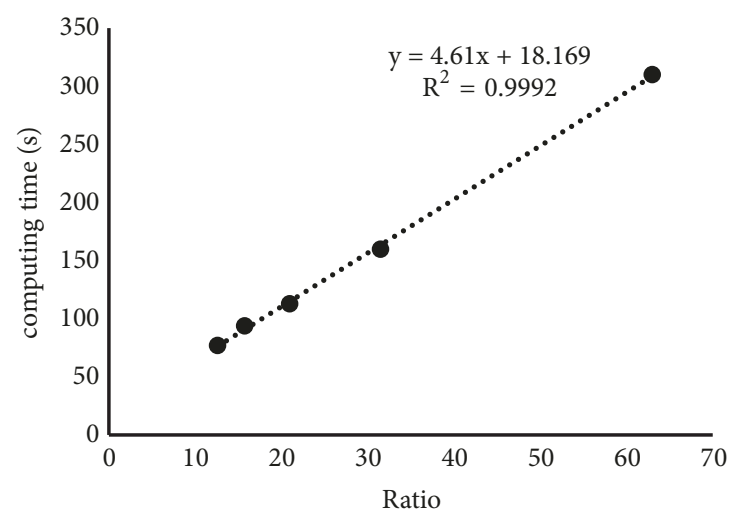

(b) Step length: $8 \mathrm{~m}$

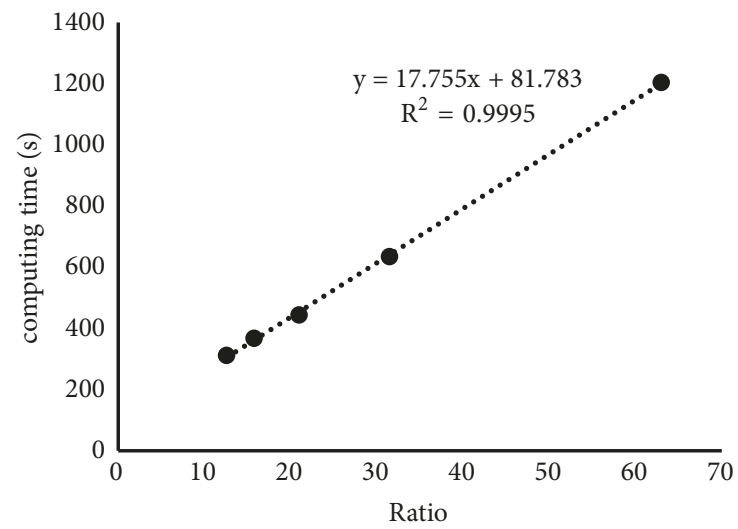

(d) Step length: $4 \mathrm{~m}$

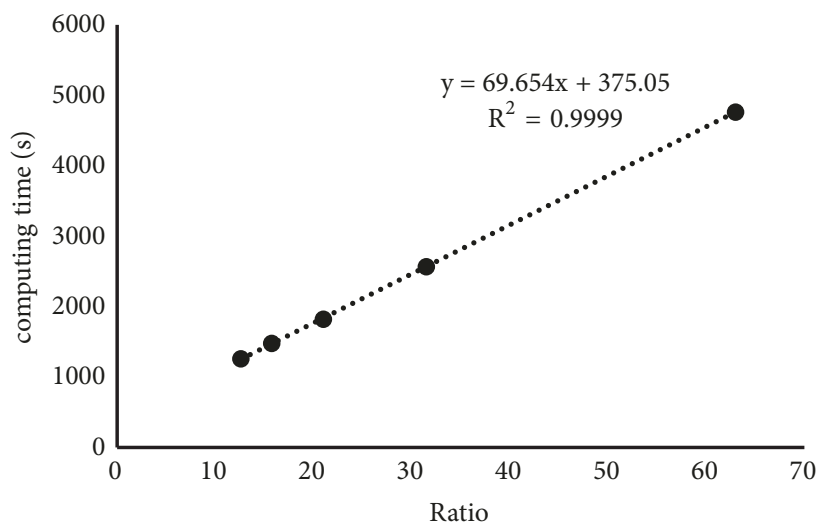

(e) Step length: $2 \mathrm{~m}$

FIgURE 6: Fitting Result of $N_{\text {block }} / N_{\text {node }}$ and $T_{\text {actual }}$.

supercomputer, and the map used in the experiment is the same map that was used in the expansibility experiment. The map is computed using one, two, and three compute nodes in the normal computer and in the supercomputer, and the result is shown in Table 3.

From Table 3, it is evident that the supercomputer is six times faster than a normal computer in terms of computation speed. As a result, it takes more than 132000 hours to calculate the same noise map of the main city zone in Guangzhou on a normal computer with a quad-core CPU because one core is used to control the other nodes. Thus, a supercomputer must be used to compute a large-scale noise map.

\section{Inhibitory Effect of Building Groups on Traffic Noise}

Building groups are the main barriers of traffic noise in urban area; thus, a scene is chosen to reflect the variation of sound field distribution causing by building groups. The scene is shown as in Figure 7 with 1034 road segments and 406 buildings.

In order to reveal the effect of building groups, a noise map without buildings of the scene is computed; then, a difference map between noise map with buildings and noise map without buildings is rendered as shown in Figure 8. 
TABLE 3: Comparison between a normal computer and a supercomputer.

\begin{tabular}{lcc}
\hline Number of compute nodes & Computing time /s & Supercomputer \\
\hline 1 & 1233 & 201 \\
2 & 670 & 109 \\
3 & 453 & 73 \\
\hline
\end{tabular}

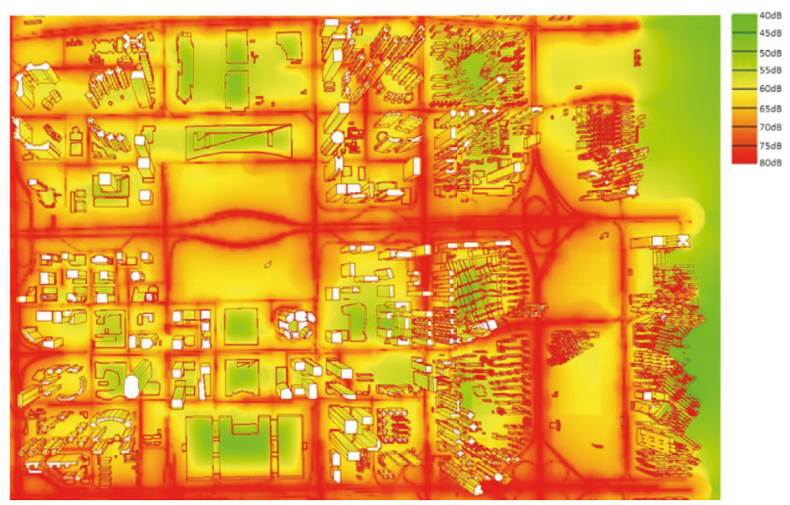

FIGURE 7: Selected scene for noise analysis.

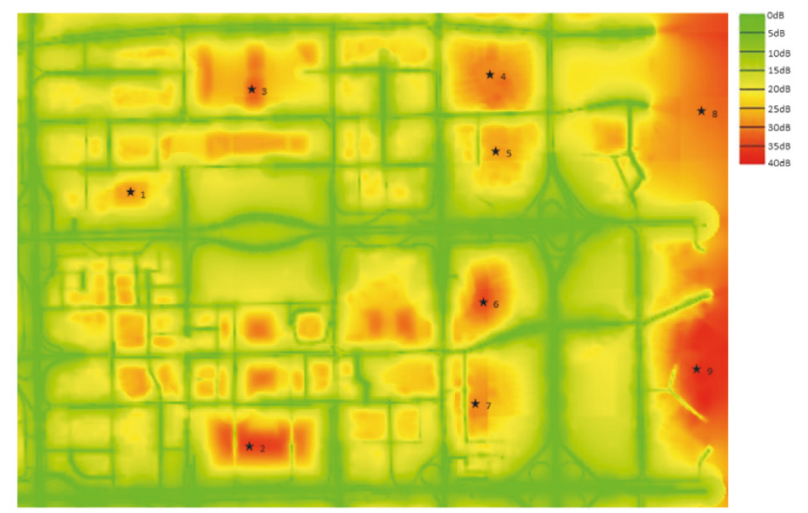

Figure 8: Difference map between noise map with or without buildings.

9 points are selected to accurately show the inhibitory effect of building groups as Table 4. From Figure 8, it is obvious that building groups have significant inhibitory effect on traffic noise; the depression of noise pressure level ranges from $0 \mathrm{~dB}$ to $40 \mathrm{~dB}$. In combination with Figure 7 , it can be found that the depression of noise pressure level is determined by the density of building groups: the denser the buildings, the greater the noise attenuation value.

Building groups not only have an inhibitory effect on horizontal noise, but also have an inhibitory effect on high buildings. Figure 9 shows the noise distribution on the building surface and black lines are noise pressure level contours. According to the distance attenuation principle of sound, the noise level at the upper floor of a building should be less than the noise level at the lower floor; however, in Figure 9, the noise level at the upper floor is higher than that
TABLE 4: Building attenuation of traffic noise.

\begin{tabular}{lc}
\hline Point number & Noise attenuation $/ \mathrm{dB}$ \\
\hline 1 & 26.2 \\
2 & 36.5 \\
3 & 32 \\
4 & 33.6 \\
5 & 25.6 \\
6 & 32.8 \\
7 & 26.1 \\
8 & 35.5 \\
9 & 37
\end{tabular}

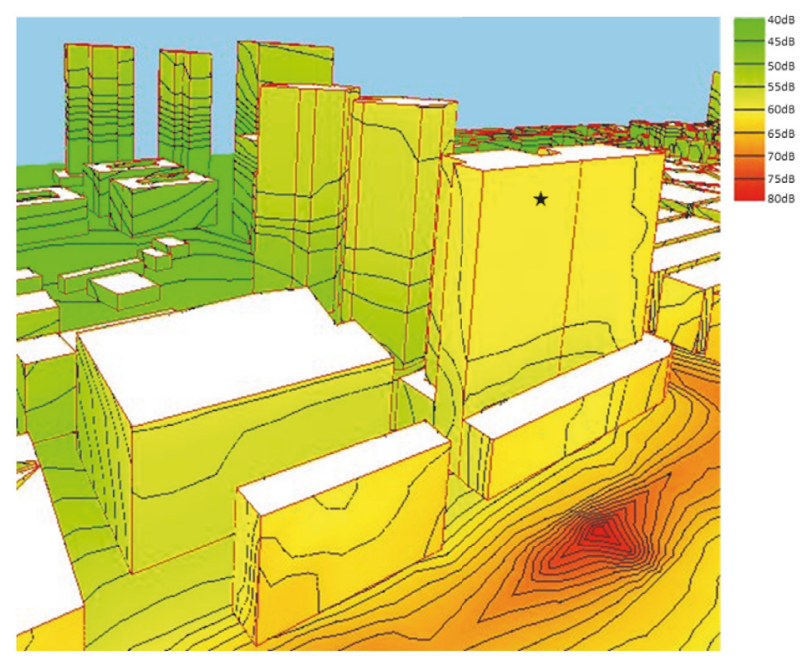

FIgURE 9: Noise distribution on the building surface.

at the lower floor in many buildings; it is because the noise at lower floor of a building will be blocked by other buildings, and the noise at upper floor is not blocked.

The noise distribution on the surface of buildings marked with asterisks is shown in Table 5. Each column in Table 5 represents each floor of the building. It can be seen from Table 5 that the noise value increases rather than decreases as the height increases.

\section{Conclusion}

A systematic methodology for computing a large-scale $3 \mathrm{D}$ traffic noise map on a supercomputer was proposed in the paper. The method covers the noise prediction model and the parallel computation algorithm used on a supercomputer. It offers a highly efficient and feasible approach for computing 
TABLE 5: Noise distribution of a building surface.

\begin{tabular}{|c|c|c|c|c|c|c|c|c|c|c|c|}
\hline Height/m & 0 & 7.5 & 15 & 22.5 & 30 & 37.5 & 45 & 52.5 & 60 & 67.5 & 75 \\
\hline \multirow{5}{*}{ Noise level of a floor/dB } & 53.36 & 55.73 & 54.02 & 54.94 & 56.31 & 57.18 & 57.47 & 57.53 & 57.55 & 57.59 & 58.35 \\
\hline & 52.8 & 53.1 & 53.41 & 54.35 & 55.72 & 56.68 & 56.84 & 56.89 & 56.92 & 57.01 & 57.55 \\
\hline & 52.73 & 52.97 & 53.21 & 54.18 & 55.51 & 56.25 & 56.65 & 56.68 & 56.7 & 56.81 & 57.46 \\
\hline & 52.76 & 52.97 & 53.19 & 54.33 & 55.62 & 56.39 & 56.78 & 56.79 & 56.81 & 56.92 & 57.31 \\
\hline & 53.08 & 53.19 & 53.45 & 54.77 & 56.06 & 56.81 & 57.3 & 57.29 & 57.31 & 57.4 & 57.68 \\
\hline
\end{tabular}

a large-scale 3D noise map. Then, to visualize the computing result, a rendering method is developed to display the rendering result of the traffic noise pollution in an urban city. Moreover, the strategy of obtaining a dynamic noise map was presented and can generate a real-time noise map in theory.

Two experiments were implemented to analyze the efficiency. The first one considered the expansibility of the parallel algorithm. When the computing scale is fixed, the computing time is positively correlated with the ratio between the number of computing blocks and the number of compute nodes: with an increase in the number of compute nodes, the computing time decreases linearly. When the computing node is fixed, with an increase in the computing scale, the ratio between the computing time and the time required for initialization remains about the same; i.e., the algorithm achieves high usage of the compute nodes. The second experiment aimed to measure the computational speed of a supercomputer. The result showed that supercomputer Tianhe- 2 is six times faster than a normal computer in terms of computing speed. Therefore, it is necessary to use a supercomputer to compute a large-scale noise map.

In addition, a scenario was chosen to analyze the impact of building groups on traffic noise. The results showed that buildings have a significant inhibitory effect on traffic noise, and due to the sheltering effect of buildings, the noise pressure level at the lower floor is lower than the upper floor in some buildings.

\section{Software Availability}

All software used in the paper can be requested from the corresponding author by email and will be provided free of charge. Program for the parallel computing of noise map is coded in $\mathrm{C}++$ and applied on supercomputer or computer with multicore and application for visualization of the computing result is coded in C\#. The supercomputer used in the paper is Tianhe-2, and a normal computer with a quad-core CPU (Inter Core i5. 3.30GHZ) and $16 \mathrm{~GB}$ of RAM is used for comparison with the supercomputer in Section 6.2. The dataset for testing the parallel algorithm can be provided in XML file and dataset for visualization noise map can be provided in text files. All files are available from the corresponding author too.

\section{Data Availability}

The data used to support the findings of this study are available from the corresponding author upon request.

\section{Conflicts of Interest}

The authors declare that they have no conflicts of interest.

\section{Acknowledgments}

This work is supported by the National Natural Science Foundation of China (no. 11574407), the Science and Technology Planning Project of Guangzhou City, China (no. 201704020142), and the Joint Foundation for SuperComputing Science Applied of NSFC and Guangdong (no. 2015_515).

\section{References}

[1] E. U. Directive, "Directive 2002/49/EC of the European parliament and the Council of 25 June 2002 relating to the assessment and management of environmental noise," Journal of the European Communities, L, vol. 189, no. 12, pp. 0012-0026, 2002.

[2] M. G. Smith, I. Croy, M. Ögren, O. Hammar, E. Lindberg, and K. Persson Waye, "Physiological effects of railway vibration and noise on sleep," The Journal of the Acoustical Society of America, vol. 141, no. 5, pp. 3262-3269, 2017.

[3] K. V. Weyde, N. H. Krog, and B. Oftedal, "Nocturnal road traffic noise exposure and childrens sleep duration and sleep problems," in Proceedings of the Nocturnal road traffic noise exposure and children's sleep duration and sleep problems. International Journal of Environmental Research Public Health, pp. 491-502, 2017.

[4] M. Sørensen, M. Hvidberg, Z. J. Andersen et al., "Road traffic noise and stroke: A prospective cohort study," European Heart Journal, vol. 32, no. 6, pp. 737-744, 2011.

[5] K.-B. Min and J.-Y. Min, "Exposure to environmental noise and risk for male infertility: A population-based cohort study," Environmental Pollution, vol. 226, pp. 118-124, 2017.

[6] P. H. T. Zannin and D. P. Z. Zwirtes, "Evaluation of the acoustic performance of classrooms in public schools," Applied Acoustics, vol. 70, no. 4, pp. 626-635, 2009.

[7] W. Babisch, W. Swart, D. Houthuijs et al., "Exposure modifiers of the relationships of transportation noise with high blood pressure and noise annoyance," The Journal of the Acoustical Society of America, vol. 132, no. 6, pp. 3788-3808, 2012.

[8] D. Botteldooren, L. Dekoninck, and D. Gillis, "The influence of traffic noise on appreciation of the living quality of a neighborhood," International Journal of Environmental Research and Public Health, vol. 8, no. 3, pp. 777-798, 2011.

[9] R. Zhang, Z. He, H. Wang, F. You, and K. Li, "Study on selftuning tyre friction control for developing main-servo loop integrated chassis control system," IEEE Access, vol. 5, pp. 66496660, 2017. 
[10] Y. Liu, B. Xia, C. Cui, and M. Skitmore, "Community response to construction noise in three central cities of Zhejiang province, China," Environmental Pollution, vol. 230, pp. 1009-1017, 2017.

[11] P. Gagliardi, L. Fredianelli, D. Simonetti, and G. Licitra, "ADS$\mathrm{B}$ system as a useful tool for testing and redrawing noise management strategies at Pisa Airport," Acta Acustica united with Acustica, vol. 103, no. 4, pp. 543-551, 2017.

[12] K. Vogiatzis and N. Remy, "Soundscape design guidelines through noise mapping methodologies: An application to medium urban agglomerations," Noise Mapping, vol. 4, no. 1, pp. $1-19,2017$.

[13] M. Ausejo, M. Tabacchi, M. Recuero, C. Asensio, R. Pagán, and I. Pavón, "Design of a noise action plan based on a road traffic noise map," Acta Acustica united with Acustica, vol. 97, no. 3, pp. 492-502, 2011.

[14] A. Ruiz-Padillo, A. J. Torija, Á. Ramos-Ridao, and D. P. Ruiz, "A methodology for classification by priority for action: Selecting road stretches for network noise action plans," Transportation Research Part D: Transport and Environment, vol. 29, pp. 66-78, 2014.

[15] D. Naish, "A method of developing regional road traffic noise management strategies," Applied Acoustics, vol. 71, no. 7, pp. 640-652, 2010.

[16] H. C. Borst and H. M. E. Miedema, "Comparison of noise impact indicators, calculated on the basis of noise maps of DENL," Acta Acustica united with Acustica, vol. 91, no. 2, pp. 378-385, 2005.

[17] M. Cai, J. Zou, J. Xie, and X. Ma, "Road traffic noise mapping in Guangzhou using GIS and GPS," Applied Acoustics, vol. 87, pp. 94-102, 2015.

[18] O. C. de Souza Neto and S. Paul, "Road traffic noise mapping in the Federal University of Santa Maria, Brazil," The Journal of the Acoustical Society of America, vol. 138, no. 3, pp. 1732-1732, 2015.

[19] M. Cai, Y. Yao, and H. Wang, "A traffic-noise-map update method based on monitoring data," The Journal of the Acoustical Society of America, vol. 141, no. 4, pp. 2604-2610, 2017.

[20] F. Alías and J. C. Socoró, "Description of anomalous noise events for reliable dynamic traffic noise mapping in real-life urban and suburban soundscapes," Applied Sciences (Switzerland), vol. 7, no. 2, 2017.

[21] D. Xie, Y. Liu, and J. Chen, "Mapping Urban environmental noise: A land use regression method," Environmental Science \& Technology, vol. 45, no. 17, pp. 7358-7364, 2011.

[22] H. Wang, H. Chen, and M. Cai, "Evaluation of an urban traffic Noise-Exposed population based on points of interest and noise maps: The case of Guangzhou," Environmental Pollution, vol. 239, pp. 741-750, 2018.

[23] J. Morel, C. Marquis-Favre, and L.-A. Gille, "Noise annoyance assessment of various urban road vehicle pass-by noises in isolation and combined with industrial noise: A laboratory study," Applied Acoustics, vol. 101, pp. 47-57, 2016.

[24] N. Fortin, E. Bocher, and J. Picaut, "An opensource tool to build urban noise maps in a GIS," in Proceedings of the Open Source Geospatial Research and Education Symposium (OGRS, pp. 226235, 2012.

[25] P. J. Sequeira Goncalves, "3D noise map of an hospital and noise sources evaluation," The Journal of the Acoustical Society of America, vol. 123, no. 5, pp. 3263-3263, 2008.

[26] A. Czyzewski, M. Szczodrak, and A. Czyżewski, "Software for Calculation of Noise Maps Implemented on Supercomputer," TASK Quarterly, vol. 13, pp. 363-377, 2009.
[27] K. Marciniuk, M. Szczodrak, and B. Kostek, "Performance of noise map service working in cloud computing environment," Archives of Acoustics, vol. 41, no. 2, pp. 297-302, 2016.

[28] H. Wang, M. Cai, and Y. Yao, "A modified 3D algorithm for road traffic noise attenuation calculations in large urban areas," Journal of Environmental Management, vol. 196, pp. 614-626, 2017.

[29] G. Licitra, M. Cerchiai, L. Teti, E. Ascari, and L. Fredianelli, "Durability and variability of the acoustical performance of rubberized road surfaces," Applied Acoustics, vol. 94, pp. 20-28, 2015.

[30] K.-Y. Ho, W.-T. Hung, C.-F. Ng, Y.-K. Lam, R. Leung, and E. Kam, "The effects of road surface and tyre deterioration on tyre/road noise emission," Applied Acoustics, vol. 74, no. 7, pp. 921-925, 2013.

[31] TOPorg, "China's Tianhe-2 is World's Top Supercomputer," Communications of the ACM, 2013.

[32] P. Bellucci, L. Peruzzi, and G. Zambon, "LIFE DYNAMAP project: The case study of Rome," Applied Acoustics, vol. 117, pp. 193-206, 2017.

[33] N. Maisonneuve, M. Stevens, M. E. Niessen, and L. Steels, "NoiseTube: Measuring and mapping noise pollution with mobile phones," Environmental Science and Engineering, vol. 2, no. 6, pp. 215-228, 2009.

[34] K. Vogiatzis and P. Kopelias, "Benefits and limitations toward a sustainable road environment during the years of economic recession," International Journal of Sustainable Development and Planning, vol. 10, no. 5, pp. 701-712, 2015.

[35] M. Cai, Z. Zhang, X. Ma, and F. Li, "Rapid updating of 3D road traffic noise maps in large cities," Noise Control Engineering Journal, vol. 64, no. 3, pp. 335-341, 2016. 


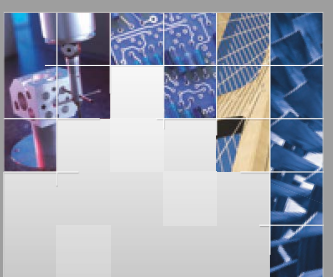

\section{Enfincering}
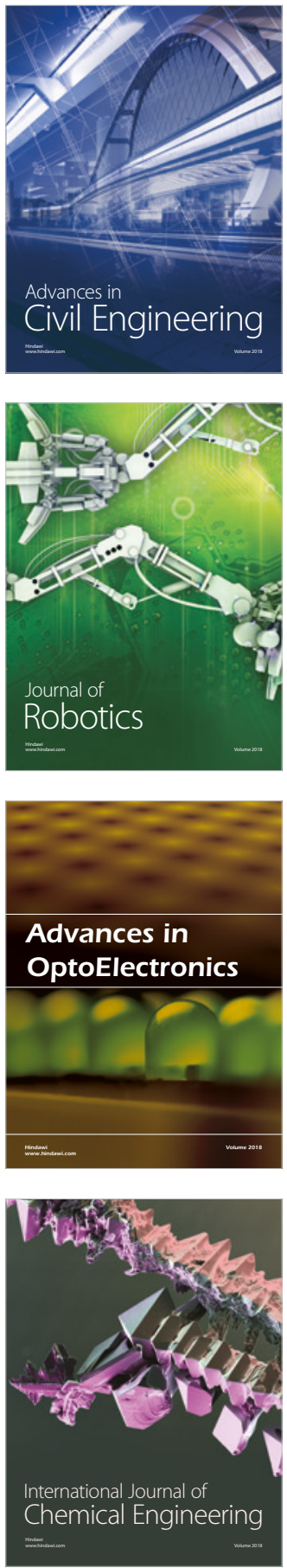

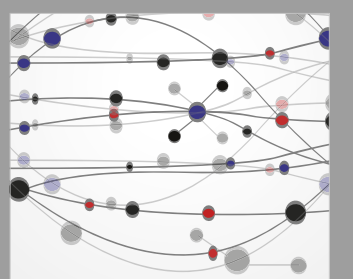

\section{Rotating \\ Machinery}

The Scientific World Journal

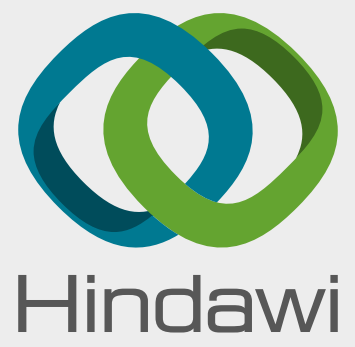

Submit your manuscripts at

www.hindawi.com
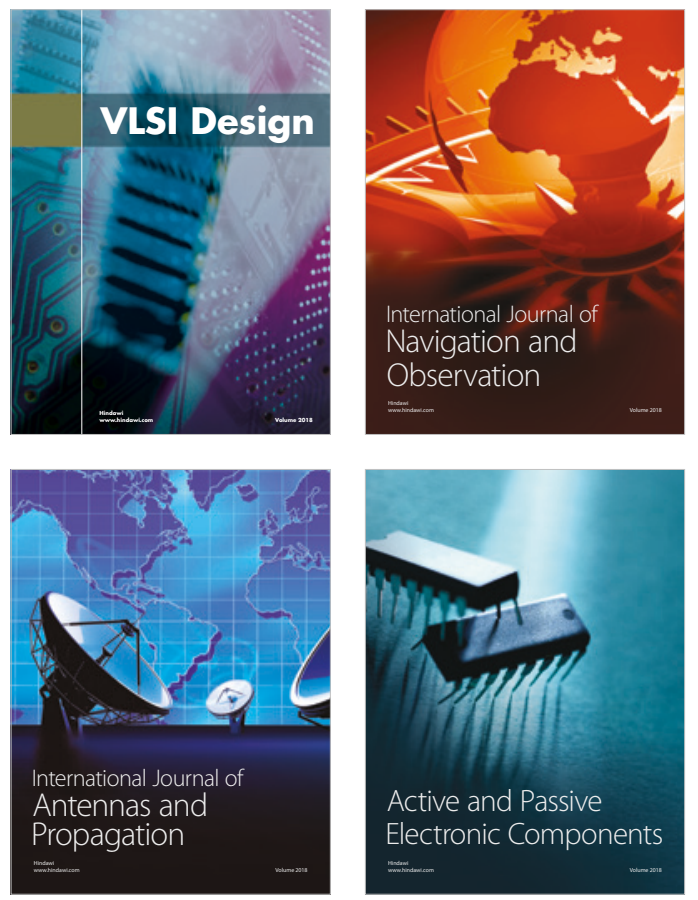
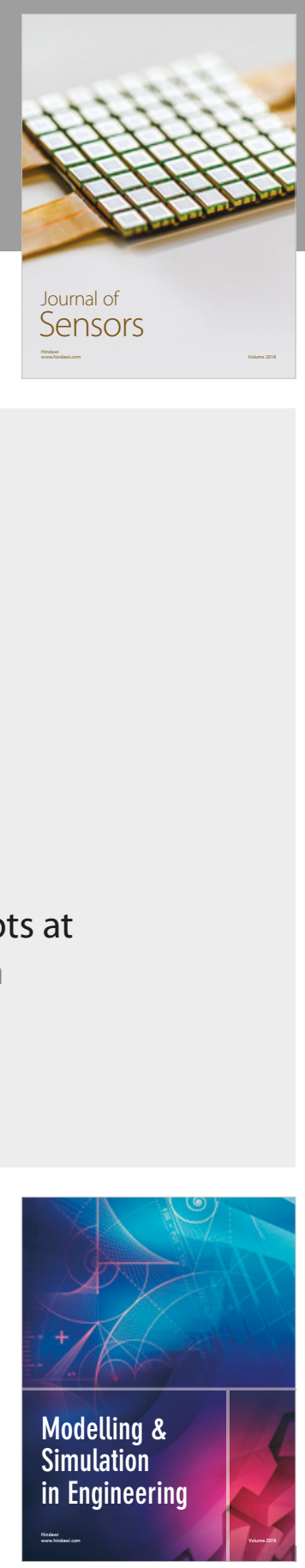

\section{Advances \\ Multimedia}
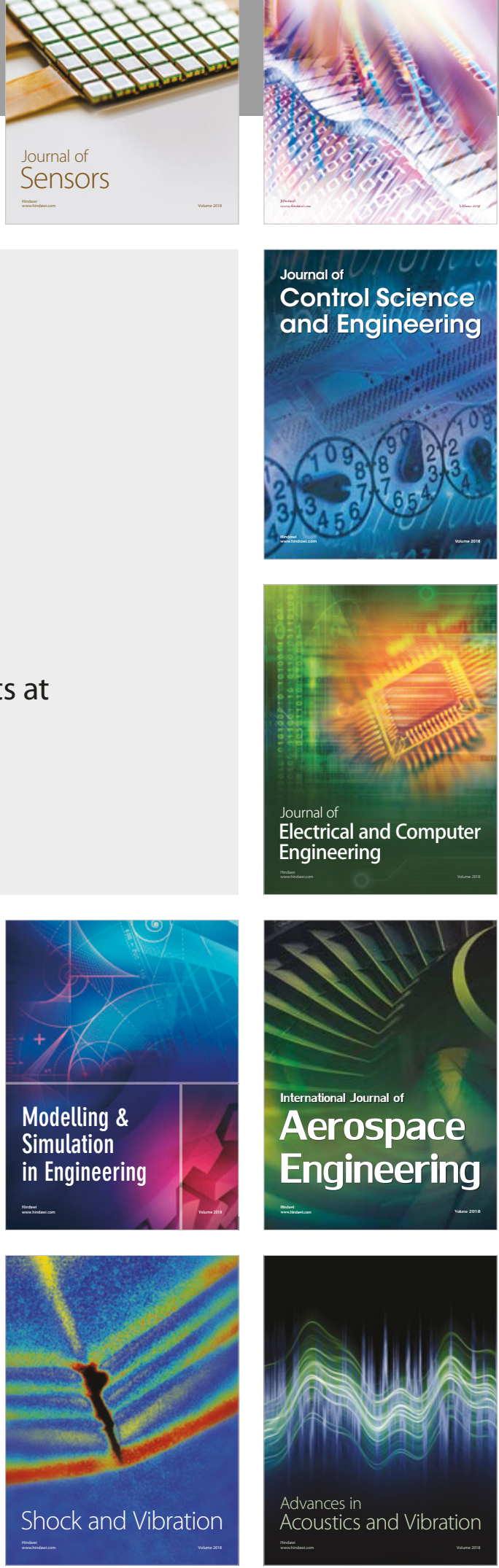